\title{
The LNGS Scientific Programme
}

\section{Ken Peach ${ }^{1}$}

Chair, LNGS Scientific Committee,

JAI, University of Oxford,

Denys Wilkinson Building, Keble Road, Oxford OXI 3RH, UK

E-mail: Ken. Peachladams-institute.ac.uk

The present and future scientific programme at the Laboratori Nazionali di Gran Sasso (LNGS) is presented and discussed.

There is something so different in Venice from any other place in the world, that you leave at once all accustomed habits and everyday sights to enter an enchanted garden.

Mary Shelley (1797-1851)

XV Workshop on Neutrino Telescopes

March 11-15, 2013

Venice, Italy

1

Speaker 


\section{Introduction}

The first experiments in deep underground laboratories took place in the 1940s and 1950s, studying cosmic rays and cosmic ray neutrinos. Since then, a number of underground laboratories have been developed, studying both cosmic rays (particularly neutrinos) and nonaccelerator physics (searches for galactic dark matter, neutrinoless double $\beta$ decay) which require screening from cosmic rays. Figure 1-a summarises the present portfolio of underground laboratories used for such experiments, and Figure 1-b shows the reduction in the cosmic ray muon flux as a function of depth. As can be seen from these figures, the Gran Sasso laboratory is, by far, the largest and although not the deepest, the surface cosmic muon flux is reduced by more than seven orders of magnitude.

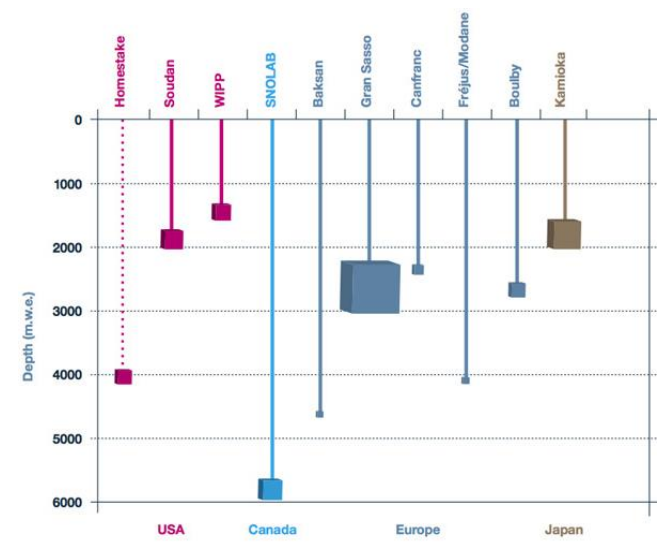

(a)

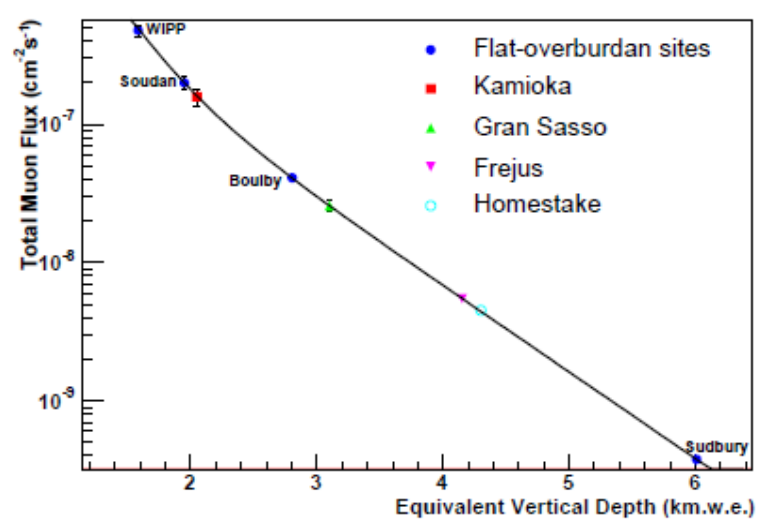

(b)

Figure 1: (a) The current underground laboratories by country, depth and size [1]. (b) The reduction in the cosmic ray muon flux as a function of depth [2].

The layout of the laboratory is shown in Figure 2. There are three main halls and several subsidiary halls, with relatively easy access from the L'Aquila-Teramo Autostrada. The experiments are at the Precision Frontier, where the emphasis is upon reducing noise, background and systematic errors. These experiments test the ingenuity of the experimentalist and the creativity of the theorist. In some senses, these experiments are an "each-way bet" - if something is found, it is a great discovery (New Physics at last) but if nothing is found, the New Physics is subjected to even more constraints, and some theories may perish.

The main programmes are discussed below

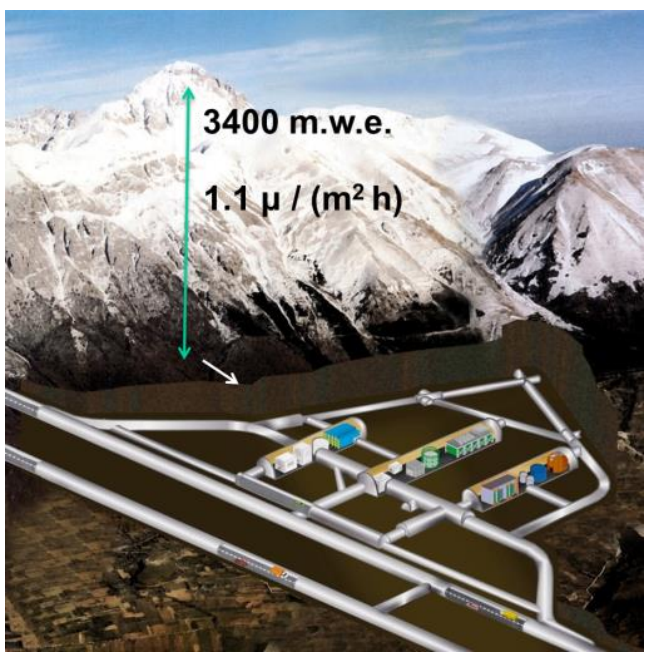

Figure 2: Layout of the Gran Sasso laboratory - neutrino physics (high energy accelerator-derived, solar and cosmogenic and double $\beta$ decay), dark matter searches, tests of fundamental principles and other science 
possibilities. In general, references are not provided - the reader is referred to talks in these proceedings by the experiments themselves, where more details can be found.

\section{Neutrino Physics}

The neutrino has been an object of fascination since Pauli's speculations [3] about its existence in the 1930s and its experimental discovery [4] by Reines and Cowan in the 1950s. In these early years, the focus of the experiments was on the nature and existence of the neutrino, including the discovery [5] of the $v_{\mu}$ in 1962. In the following 30 years, the neutrino was used as a probe to study the structure functions $\left(\mathrm{F}_{2}, \mathrm{xF}_{3} \ldots\right)$ of the nucleon and the structure of the weak interaction (neutral currents, $\sin \theta_{\mathrm{w}} \ldots$ ), but for the last 15 years or so, with the discovery of neutrino oscillations, the focus has reverted to the nature of the neutrino itself - the oscillation parameters and the absolute mass scales. What this has made clear is that the Standard Model assumption that the neutrino is strictly massless is wrong; it is difficult to add a neutrino mass term to the Standard Model Lagrangian consistently, and the features of a neutrally charged chiral fermion creates possibilities, for example Majorana fermions, that are new physics. The LNGS neutrino programme addresses many of these issues.

\subsection{High Energy Neutrinos}

The CERN Neutrinos to Gran Sasso (CNGS) beam directs high-energy, predominantly muon, neutrinos through the earth to the LNGS some $730 \mathrm{~km}$ away. The neutrino energy is well above the threshold for creating $\tau$ neutrinos, and the principal focus of the programme is to search for the rare oscillation of $\mu$-neutrinos $\left(v_{\mu}\right)$ into $\tau$ neutrinos $\left(v_{\tau}\right)$, which happens with a probability proportional to $\cos ^{4} \theta_{12} \sin ^{2} 2 \theta_{23}$ $\sin ^{2} \Delta_{32}$, although the beam allows a wider range of other neutrino measurements to be performed.

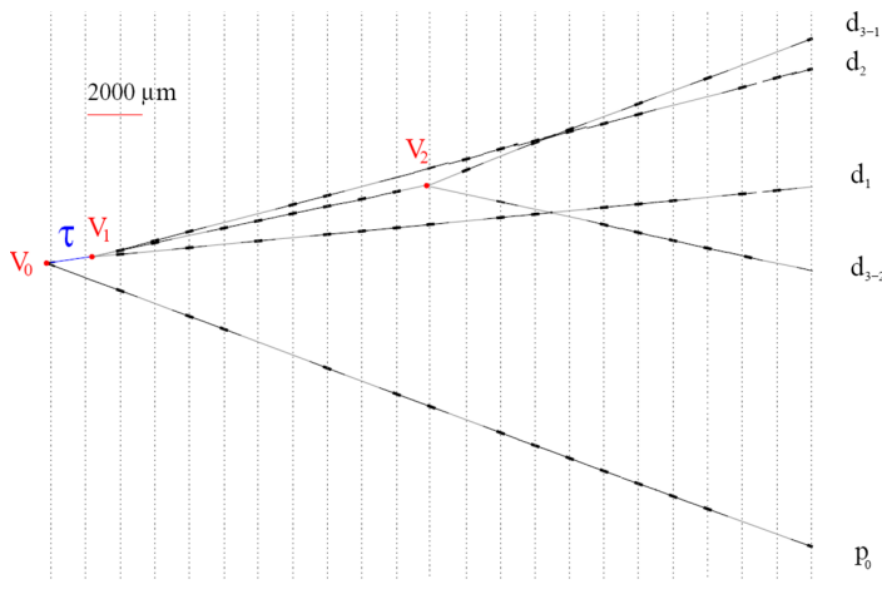

Figure 3: A $v_{\tau}$ candidate in the OPERA experiment.

The OPERA experiment consists of a large magnetised spectrometer with a huge emulsion detector (which also acts as the neutrino target) and a sophisticated emulsion manipulation and analysis system. Two candidate events for a $v_{\mu} \rightarrow v_{\tau}$ oscillation have been observed (and a third since this conference) in line with expectations - see Figure 
3 for an image of a $\tau$-neutrino candidate. The experiment has also observed candidates for sub-dominant $v_{\mu} \rightarrow v_{\mathrm{e}}$ oscillation which can be used to put further constraints on the amplitude and coupling of non-standard oscillations.

The other large experiment is ICARUS, a 770T, fully active liquid argon TPC - an "electronic bubble chamber". This gives images of extraordinary clarity, as shown in Figure 4. The detector had its commissioning run in 2010 and started data-taking later that year, and has now observed more than 4000 charged and neutral current events in the CNGS beam. The fine granularity of the liquid argon technique allows for good flavour detection; ICARUS has searched for $v_{\mathrm{e}}$ appearance in the LSND parameter space, and placed quite stringent limits on the oscillation parameters.

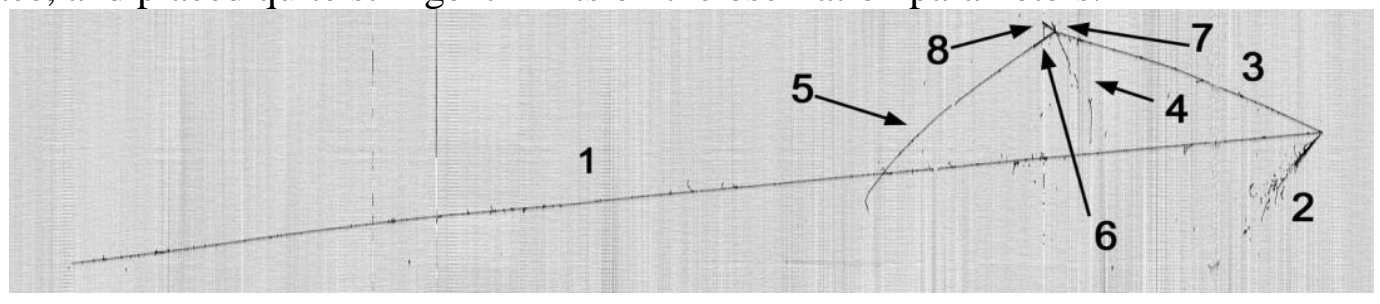

Figure 4 A neutrino event with 8 resolved tracks from ICARUS

Both experiments have verified that the neutrino velocity is consistent with expectations, as have the Borexino and LVD experiments discussed below. In addition, since the CNGS beam passes through both OPERA and LVD, it has also been possible to cross-check the relative timing of both detectors, which are now consistent.

\subsection{Solar and cosmogenic neutrinos}

Solar neutrinos from burning hydrogen in the sun provide an intense $\left(7 \times 10^{10} / \mathrm{cm}^{2} / \mathrm{s}\right)$ flux of low energy $(\sim \mathrm{MeV})$ electron neutrinos at the earth's surface. The spectral flux of neutrinos can be calculated from models of the solar fusion processes, and it has been known for more than 40 years that fewer electron neutrinos are detected than expected, and that this deficit has an energy dependence. Neutrino oscillations provide a natural explanation for these observations, and the phenomenology is now well-established and well-understood. Nevertheless, the history of the neutrino has been full of surprises so that it is prudent to check everything, just in case there is some revolution hiding in the data. Secondly, having established the properties of the neutrino, the neutrino can again be used to study something else ... in this case, precision studies of the solar fuel cycle.

The Borexino experiment consist of a large volume of liquid scintillator surrounded by an even larger water shield, monitored by photomultipliers. The low threshold means that the detector is sensitive to the $\mathrm{pp}$, pep and ${ }^{7} \mathrm{Be}$ neutrinos which have energies around an $\mathrm{MeV}$ or so, as well as the higher energy ${ }^{8} \mathrm{Be}$ neutrinos (see Figure 5). The experiment has also (at more than $3 \sigma$ ) observed a seasonal modulation of the ${ }^{7} \mathrm{Be}$ flux as expected. Remarkably, there is also firm evidence for the observation of geo-neutrinos. 


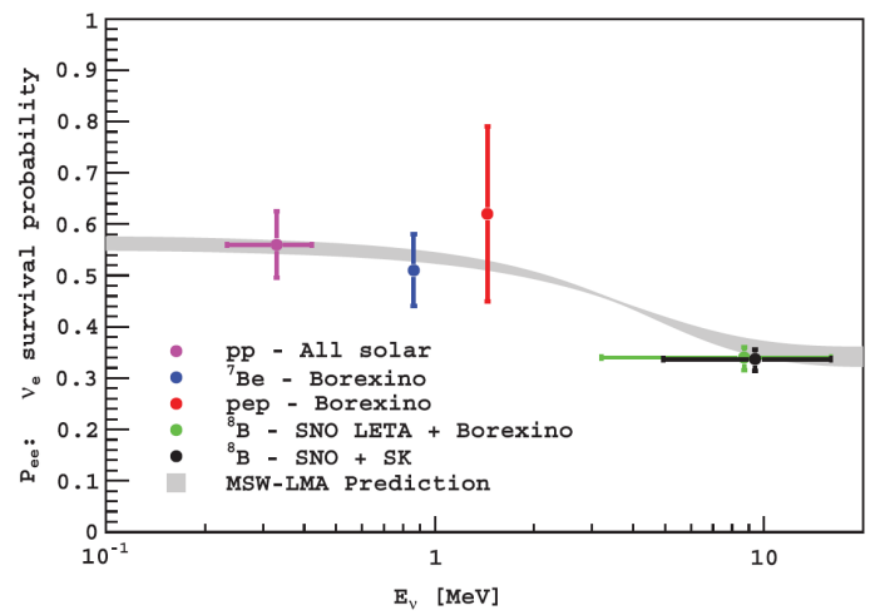

Figure 5: The Borexino data on solar neutrinos. The purple data point is from the combined Borexino and solar experiments data, the blue point is the ${ }^{7}$ Be rate, the red point is the pep rate and the black point the ${ }^{8} \mathrm{Be}$ rate. The grey line is the expected survival probability from the solar model and the neutrino oscillation parameters.

The LVD (Large Volume Detector 1kT) has been operating since 1992 with high efficiency, searches for the neutrino pulse from a supernova collapse; it is linked in to the SNEWS network. The detector is very stable, with a high availability - see Figure 6. Since 2001 LVD has measured an annual modulation of the muon flux through the detector over eight cycles, which correlates well with the measured radon concentration.

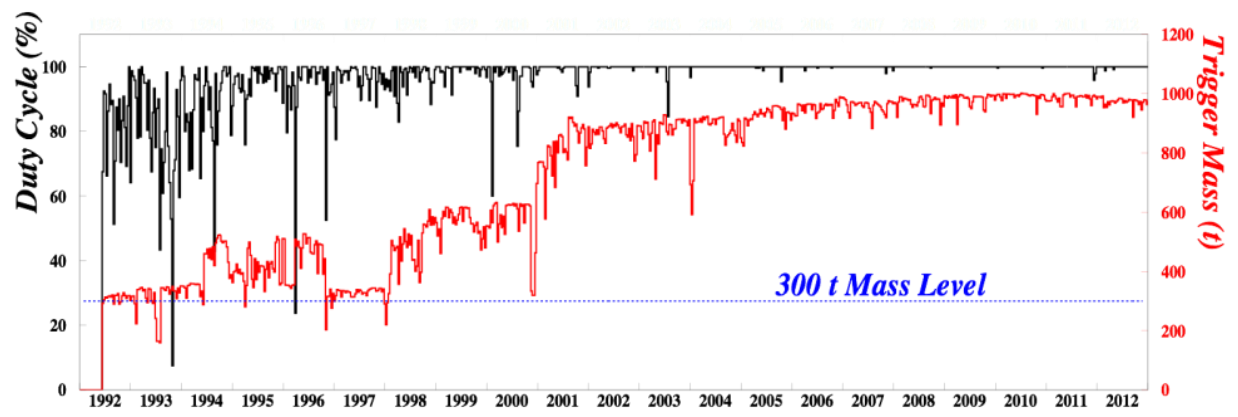

Figure 6: The availability and target mass of the LVD detector since 1992

When the present Borexino programme is complete, there is a proposal (SOX - Short

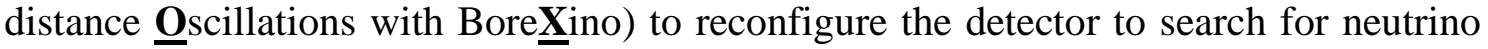
oscillations from low energy neutrinos and/or antineutrinos on a short (few metre) baseline. There are three options $-\mathrm{a}{ }^{51} \mathrm{Cr}$ neutrino source placed outside the detector, and a ${ }^{144} \mathrm{Ce}$ antineutrino source placed in the water shield and/or the scintillator liquid.

\subsection{Neutrinoless double beta decay}

If the neutrino has a Majorana component (i.e. the neutrino is its own antiparticle), the rare process whereby two successive $\beta$-decays occur can either emit two (anti)neutrinos $(2 v \beta \beta)$ or no neutrinos $(0 v \beta \beta)$, the rate being determined by the appropriate weighted combination of the mass eigenstate values. There are about a dozen suitable nuclei where this process might occur, and the signature (two electrons with a sharp combined energy $\left.Q_{\sigma_{\nu \beta \beta}}=m(A, Z)-m(A, Z \pm 2)-2 m_{e}\right)$ is distinctive; however, the lifetimes 
are long ( $>10^{22}$ years typically) and backgrounds (from the normal double $\beta$ decay and from radioactivity) need to be tightly controlled. While the first observation of $0 v \beta \beta$ will, of course, be a major discovery (a Majorana particle), extracting a precise value for the mass parameter will probably require its observation in several nuclei, to reduce the uncertainty arising from the nuclear matrix elements.

The LNGS programme includes four experiments looking for $0 v \beta \beta$ - GERDA (which uses ${ }^{76} \mathrm{Ge}$ as the target nucleus, with $\left.\mathrm{Q}_{0 \vee \beta \beta}=2.039 \mathrm{MeV}\right), \mathrm{CUORE}\left({ }^{130} \mathrm{Te}\right.$, $\mathrm{Q}_{0 v \beta \beta}=2.528 \mathrm{MeV}$ ), COBRA (several potential channels in CdZnTe, but focussing on $\left.{ }^{116} \mathrm{Cd}, \mathrm{Q}_{0 \vee \beta \beta}=2.813 \mathrm{MeV}\right)$ and LUCIFER $\left({ }^{82} \mathrm{Se}, \mathrm{Q}_{0 \vee \beta \beta}=2.997 \mathrm{MeV}\right)$. GERDA uses an array of enriched germanium crystals surrounded by a large water shield, and have accumulated more than $15 \mathrm{~kg}$.y of data. They have measured the normal $2 v \beta \beta$ half-life to be $1.84^{+0.09+0.11}-0.08-0.06 \times 10^{21} \mathrm{y}$, and have a background in the signal region of about 0.02 counts per $\mathrm{keV}$ per $\mathrm{kg} \cdot \mathrm{y}$; the goal for the second phase is to reduce this to less than 0.001 counts per $\mathrm{keV}$ per $\mathrm{kg} \cdot \mathrm{y}$. GERDA hope to release the results of the first phase later this year'. CUORE is in construction, and will eventually have $9880.75 \mathrm{~kg} \mathrm{TeO}_{2}$ crystals in a large low-noise $6 \mathrm{mK}$ cryostat, with more than $200 \mathrm{~kg}$ of ${ }^{130} \mathrm{Te}$. A demonstrator phase (CUORE-0) has a single tower of 13 planes with 4 crystals in each plane which will be deployed in an existing cryostat. This is being used to develop the assembly techniques for the full experiment, but will be capable of delivering an interesting result (limit around $10^{25} \mathrm{y}$ ) after two years of data taking. Unlike most of the ov $\beta \beta$ searches, COBRA operates at room temperature, using CdZnTe, a commercially available semiconductor, which provides both the target nuclei and the pixelated tracking detector. The detector uses arrays (somewhat like a chess board) of $641 \mathrm{~cm}^{3}$ detectors, with the electron tracks extending for 1-2 $\mathrm{mm}$ ( $\sim 12$ pixels). Prototype arrays of 32 detectors have been tested, with reasonably good results. LUCIFER uses a scintillating ZnSe bolometer, using enriched selenium. A tower of 32-40 crystals will be placed, 4 crystals per floor, in a cryostat and hopes to take advantage of the very high $\mathrm{Q}$ value to observe the decay. At present, the focus is on the crystal procurement and production - this is a rather specialised process which needs specialist manufacture. The experiment should start taking data in 2015.

\section{Dark Matter Searches}

The second major strand of the LNGS scientific programme is the search for galactic dark matter. The astrophysical and cosmological evidence for the existence of dark matter is strong, from the original observation of the rotation velocities of stars in spiral galaxies to phenomena such as the dynamics of colliding galaxies and the evolution of the early universe. While there may be (plausible or implausible) alternative explanations for each of these phenomena, they are all addressed by the appeal to dark matter; application of Occam's Razor implies that dark matter should exist, and that its effects should be directly observable with sensitive enough searches if the nature of dark matter is a weakly interacting massive particle (WIMP). There are, of course, candidates from extensions to the Standard Model of Particles and their Interactions that could satisfy the dark matter requirements, supersymmetry being perhaps the most

\footnotetext{
${ }^{2}$ This has now been published, with the half-life being greater than $3 \times 10^{25}$ y at $90 \%$ C.L. (M. Agostini et al, Phys. Rev. Lett. 111, 122503 (2013))
} 
popular. The advantage of analysing the prospects for dark matter discovery within the context of a theory such as supersymmetry is that it provides an estimate of the upper limit (as a function of the WIMP mass) of the cross-section that is expected, currently indicating small cross-sections $\left(<10^{43} \mathrm{~cm}^{2}\right)$ for WIMP masses of several hundred $\mathrm{GeV}$ (see Figure 7).

As with $0 v \beta \beta$, the first observation of galactic dark matter will be a major discovery (and indeed, there are some claims - not universally accepted - of signals or potential signals) but given the difficulty of the experiments and the need to exercise firm control backgrounds and systematic errors, confirmation from independent experiments using different techniques will be needed, and to enable information about the nature of the dark matter (mass, interactions, possibly spin) top be extracted. There are four major experiments searching for dark matter at LNGS.

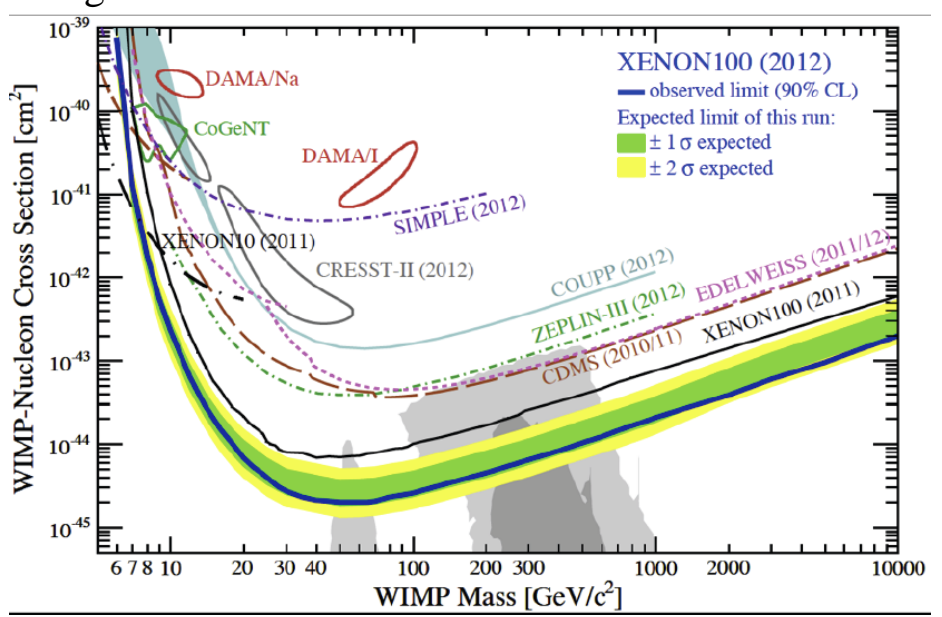

Figure 7 Summary of the dark matter searches (March 2013) showing the latest XENON-100 limit and a selection of other limits and claims. The shaded areas indicate possible regions for a variety of supersymmetric models.

The DAMA/LIBRA experiment looks for the annual modulation expected as the Earth moves through the dark matter cloud by studying the rate in radiologically pure, well-shielded Sodium Iodide crystals. Indeed DAMA/LIBRA have observed such a modulation, now over more than 13 cycles, with a phase consistent with that expected from a galactic dark matter signal. If interpreted as evidence for dark matter, it indicates a relatively light WIMP mass $(10-100 \mathrm{GeV})$ and a large cross-section $\left(>10^{41} \mathrm{~cm}^{2}\right)-$ see Figure 7 - but this is not supported by other experiments ${ }^{3}$.

CRESST uses cryogenic calcium tungstate (and one zinc tungstate) crystals with photon and phonon detectors - the phonon channel gives a precise measurement of the energy and the photon signal provides discrimination between different types of particle interaction, specifically to reject electromagnetic background. An analysis of $8 \mathrm{CaWO}_{4}$ crystals with $730 \mathrm{~kg}$-days exposure observed 67 events, with a claim (see Figure 7) that this indicated an excess of either $29.4^{+8.6}{ }_{-7.7}$ or $24.2^{+8.1}-7.2$ events, corresponding to a low WIMP mass $(25.3$ or $11.6 \mathrm{GeV})$ and a high cross-section $\left(>10^{-42} \mathrm{~cm}^{2}\right.$ or $\left.10^{-41} \mathrm{~cm}^{2}\right)$, although this claim is controversial.

\footnotetext{
${ }^{3}$ The CoGENT collaboration have reported evidence for an annual modulation using low-noise germanium detectors[C.E. Aalseth et al, Phys. Rev. Lett. (2011) 106 1331301], but with parameters that do not agree well with DAMA/LIBRA.
} 
The XENON series of experiments (XENON-10, XENON-100 and XENON-1T) uses dual phase (liquid-gas) Xenon as both target and detector, with both charge and light detection to provide energy and position reconstruction and background rejection. The fiducial volume is self-shielded and the technique has good event detection and discrimination. The current world-leading analysis comes from the XENON-100 100kg run (see Figure 7). The XENON-1T detector will have a fiducial mass of about 1T of liquid Xenon and be surrounded by an active water shield, and is under construction. The sensitivity limit should be better than $10^{-46} \mathrm{~cm}^{2}$ for a range of WIMP masses from about $20 \mathrm{GeV}$ to more than $1 \mathrm{TeV}$.

An alternative noble liquid dark matter detector uses two-phase liquid argon, and the Darkside collaboration is constructing a large volume detector (fiducial mass about 3.3T), surrounded by a large active water shield (actually a test facility that was used for Borexino). A small (50kg) prototype (Darkside-50) has operated and produced excellent results (a high light yield of 8.9 photoelectrons per $\mathrm{keV}$ ). A special feature of the Darkside experiment is the pioneering use of "underground argon", which is uncontaminated by radioactive debris from nuclear testing - about $0.5 \mathrm{~kg}$ of argon is produced per day, with an activity that is about two orders of magnitude less than commercially available argon. Darkside-50 has a sensitivity of about $2 \times 10^{-45} \mathrm{~cm}^{2}$, and the complete Darkside detector should have a limit of around $2 \times 10^{-47} \mathrm{~cm}^{2}$.

As can be seen from Figure 7, although there are indications of dark matter signals ${ }^{4}$ there is no consistency between them. What is needed is more, and better, experiments (ugly facts), so that "the great tragedy of Science: the slaying of a beautiful hypothesis by an ugly fact [6]" can be re-enacted.

\section{Tests of Fundamental Principles}

It is important in science to be sceptical and to challenge the accepted orthodoxy. The probability that some cherished principle might be found wanting may be vanishingly small, but the consequences if the principle is violated, however weakly, are correspondingly profound. In contrast to the experiments described above, which need large teams of scientists to design, build, operate and analyses, such experiments are often pursued by a small group with considerable zeal. The classic examples are parity and $\mathrm{CP}$ violation, both of which could have been easily discovered years before they were, but were not because no-one thought it worth investigating. What these experiments share with the neutrino and dark matter experiments is the attention to detail, the control of systematics and backgrounds, and the application of systematic doubt.

The VIP experiment (VIolation of the PEP) looks for violation of the Pauli Exclusion Principle (PEP) by search for forbidden (2p-1s) transitions in an electric current circulating in a copper conductor. The normal $2 \mathrm{p}-1 \mathrm{~s}$ transition can, of course, take place if there is a vacancy in the $\mathrm{n}=1$ level, and has a transition energy of $8.04 \mathrm{keV}$. However, if there is a small PEP-violating amplitude, there is a possible transition to the ground state with 3 electrons, and the transition energy is only $7.7 \mathrm{keV}$. There are several assumptions that underlie the PEP, and violation of the PEP could imply

\footnotetext{
${ }^{4}$ and since this conference the situation has been further complicated by the observation of 3 events in the signal region by the CDMS experiment R. Agnesi et al http://arxiv.org/abs/1304.4279 (2103)
} 
violation of one or more of the following; CPT, locality, Lorentz invariance, extra space dimensions, discrete space and/or time and non-commutative space-time. The experiment has already taken data, and obtained a limit for $\beta$ (which represents the PEPviolating amplitude) of $1 / 2 \beta^{2}<3 \times 10^{-29}$, where $1 / 2 \beta^{2}$ is the probability for an electron to make the transition. The experiment has been redesigned and upgraded to gain two orders of magnitude improvement in sensitivity after 4 years of data taking.

The GINGER ( Gyroscopes IN GEneral Relativity) experiment uses an array of highprecision optical ring lasers. These allow, in principle, the Earth's rotation (angular velocity vector) to be measured with very high precision. There is an influence through general relativity on the rate of precession due to the angular momentum of nearby objects which define the local frame of reference, known as the Lense-Thirring effect. This influence can be measured using the Sargnac effect in an interferometer, where two beams contra-rotating in a ring exhibit a phase shift related to the angular momentum. Figure 8 shows the order of magnitude contributions to the measured angular rotation. This requires a delicate setup of the equipment; a pathfinder project is underway in LNGS to evaluate the options.

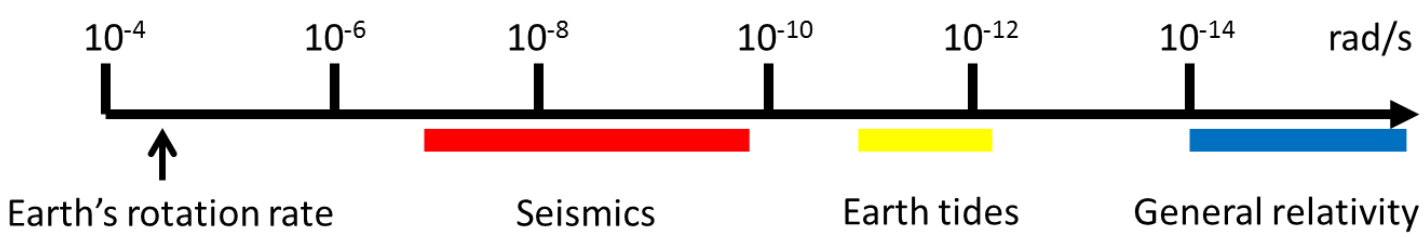

Figure 8 The contributions to the measurement of the Earth's angular velocity.

\section{Other science}

The existence of a large, well-appointed and easily accessible (well, relatively easily) underground laboratory creates opportunities for other science well beyond the original scientific programme, all of which require, for one reason or another, respite from the cosmic rain.

The LUNA and LUNA-MV programmes study exceptionally rare nuclear processes which are of particular relevance to astrophysics (both nucleosynthesis and the evolution of stars, including supernovae). The main asset is a low-energy accelerator capable of accelerating ions to modest energies $(50 \mathrm{kV}$ in phase $1,400 \mathrm{kV}$ in phase 2 now in operation, and eventually to $3.5 \mathrm{MV}$ energies in the phase under consideration). The underground location is required to take advantage of the six (three) orders of magnitude reduction in the muon (neutron) fluxes. An extensive range of reactions has already been studied: ${ }^{3} \mathrm{He}\left({ }^{3} \mathrm{He}, 2 \mathrm{p}\right){ }^{4} \mathrm{He},{ }^{3} \mathrm{He}\left({ }^{4} \mathrm{He}, \gamma\right){ }^{7} \mathrm{Be}$ and ${ }^{14} \mathrm{~N}(\mathrm{p}, \gamma){ }^{15} \mathrm{O}$ (important for the modelling of the sun); ${ }^{14} \mathrm{~N}(\mathrm{p}, \gamma){ }^{15} \mathrm{O}$ (for determining the age of globular clusters); ${ }^{15} \mathrm{~N}(\mathrm{p}, \gamma){ }^{16} \mathrm{O},{ }^{17} \mathrm{~N}(\mathrm{p}, \gamma){ }^{18} \mathrm{O}$ and ${ }^{25} \mathrm{Mg}(\mathrm{p}, \gamma){ }^{26} \mathrm{Al}$ (light element nucleosynthesis); and ${ }^{2} \mathrm{H}(\mathrm{a}, \gamma){ }^{6} \mathrm{Li},{ }^{3} \mathrm{He}\left({ }^{4} \mathrm{He}, \gamma\right){ }^{7} \mathrm{Be}$ and ${ }^{2} \mathrm{H}(\mathrm{p}, \gamma){ }^{3} \mathrm{He}$ (Big Bang nucleosynthesis). There is a series of reactions under study, continuing the light element nucleosynthesis (e.g. ${ }^{17} \mathrm{O}(\mathrm{p}, \alpha){ }^{14} \mathrm{~N}$, $\left.{ }^{22} \mathrm{Ne}(\mathrm{p}, \gamma){ }^{23} \mathrm{Na},{ }^{23} \mathrm{Na}(\mathrm{p}, \gamma){ }^{24} \mathrm{Mg},{ }^{18} \mathrm{O}(\mathrm{p}, \gamma){ }^{19} \mathrm{~F},{ }^{18} \mathrm{O}(\mathrm{p}, \alpha){ }^{15} \mathrm{~N}\right)$, helium burning and stellar evolution $\left({ }^{12} \mathrm{C}(\alpha, \gamma){ }^{16} \mathrm{O}\right)$ and s-process nucleosynthesis $\left({ }^{13} \mathrm{C}(\alpha, \mathrm{n}){ }^{16} \mathrm{O},{ }^{22} \mathrm{Ne}(\alpha, \mathrm{n}){ }^{25} \mathrm{Mg}\right)$. LUNA-MV is funded for a $3.5 \mathrm{MV}$ single-ended positive ion accelerator, which will 
allow the further study of key s-process reaction, relevant to helium burning, which takes place at higher temperatures than hydrogen burning.

The ERMES-WOLRD project is a large, multi-disciplinary international programme of research addressing environmental issues from climate change, the internal structure of the Earth and global geodynamic processes to primary resource (water) management. The principal tool, and the need for an underground location, arises from the common use of radionuclide markers which requires an ultra-low background environment.

Finally, there is interest in studying the behaviour of biological materials in an ultralow radiation environment. There are tantalising results from in vitro experiments which call into question the assumptions implicit in the Linear No Threshold (LNT) model of radiation damage, which could have important implications for radiation protection and nuclear medicine. The LNT model assumes that the damage to cells DNA is strictly proportional to dose, implying that there is no completely "safe dose" of radiation (although the risk of harm may be vanishingly small). However, it might also be that very low doses could be significantly more, or less, damaging than the LNT model implies. More work is needed, and there are few environments where this can be done. (Increasing the dose and looking at the linearity of the response unfortunately does not provide a robust answer, for obvious reasons.)

\section{Summary}

The scientific programme is world-leading and addresses important scientific issues in neutrino physics, dark matter searches, the foundations of physics and cosmology and potentially the environment and health. It is a national laboratory and an international asset.

\section{References}

[1] Plot adapted from http://www.deepscience.org/contents/facilities.shtml

[2] A. Hime and D-M Mei, Muon-induced background study for underground laboratories, Phys. Rev. D73 (2006) 053004

[3] Letter from W. Pauli to the "Liebe Radioaktive Damen und Herren", $4^{\text {th }}$ December 1930.

[4] C. L. Cowan, Jr., F. Reines, F. B. Harrison, H. W. Kruse and A. D. McGuire, Detection of the Free Neutrino: A Confirmation, Science 124 (1956) 103

[5] G. Danby, J.-M. Gaillard, K. Goulianos, L. M. Lederman, N. B. Mistry, M. Schwartz, J. Steinberger, Observation of high-energy neutrino reactions and the existence of two kinds of neutrinos, Phys. Rev. Lett. (1962) 936

[6] T.H. Huxley (1825-1895) 